\title{
MRSA prevalence in european healthcare settings: a review
}

Madeleine Dulon ${ }^{1 *}$, Frank Haamann ${ }^{1}$, Claudia Peters ${ }^{2}$, Anja Schablon $^{2}$ and Albert Nienhaus ${ }^{2}$

\begin{abstract}
Background: During the past two decades, methicillin-resistant Staphylococcus aureus (MRSA) has become increasingly common as a source of nosocomial infections. Most studies of MRSA surveillance were performed during outbreaks, so that results are not applicable to settings in which MRSA is endemic. This paper gives an overview of MRSA prevalence in hospitals and other healthcare institutions in non-outbreak situations in Western Europe.

Methods: A keyword search was conducted in the Medline database (2000 through June 2010). Titles and abstracts were screened to identify studies on MRSA prevalence in patients in non-outbreak situations in European healthcare facilities. Each study was assessed using seven quality criteria (outcome definition, time unit, target population, participants, observer bias, screening procedure, swabbing sites) and categorized as 'good', 'fair', or 'poor'.

Results: 31 observational studies were included in the review. Four of the studies were of good quality. Surveillance screening of MRSA was performed in long-term care (11 studies) and acute care (20 studies). Prevalence rates varied over a wide range, from less than $1 \%$ to greater than $20 \%$. Prevalence in the acute care and long-term care settings was comparable. The prevalence of MRSA was expressed in various ways - the percentage of MRSA among patients (range between 1\% and 24\%), the percentage of MRSA among S. aureus isolates (range between 5\% and 54\%), and as the prevalence density (range between 0.4 and 4 MRSA cases per 1,000 patient days). The screening policy differed with respect to time points (on admission or during hospital stay), selection criteria (all admissions or patients at high risk for MRSA) and anatomical sampling sites.
\end{abstract}

Conclusions: This review underlines the methodological differences between studies of MRSA surveillance. For comparisons between different healthcare settings, surveillance methods and outcome calculations should be standardized.

\section{Background}

Staphylococcus aureus (S. aureus) is a versatile human pathogen that causes diseases ranging from relatively mild infections of the skin and soft tissue to life-threatening sepsis [1]. The emergence of strains resistant to methicillin and other antimicrobial agents has become a major concern, especially in the hospital environment, because of the higher mortality due to systemic methicillin-resistant Staphylococcus aureus (MRSA) infections [2]. Tiemersma et al. [3] have shown significant increases in methicillin resistance in clinical strains of $S$. aureus isolates between 1999 and 2002 in European countries,

\footnotetext{
* Correspondence: madeleine.dulon@bgw-online.de

'Institution for Statutory Accident Insurance and Prevention in the Health and Welfare Services. Department of Occupational Health Research,

Pappelallee 33/35/37, 22089 Hamburg, Germany

Full list of author information is available at the end of the article
}

particularly Belgium, Germany, Ireland, the Netherlands and the United Kingdom. MRSA prevalence varied widely, from $<1 \%$ in northern Europe to $>40 \%$ in southern and western Europe [3]. As the prevalence of healthcare-associated infections (HAIs) caused by multidrugresistant organisms continues to increase [4], it seems essential to prevent MRSA transmission and reduce the number of MRSA HAIs. It is also important for healthcare workers that MRSA rates should be controlled, as a recently published review has shown that the average MRSA carrier rate in healthcare workers is $4.6 \%$, and that about $5.1 \%$ of these carriers had symptomatic MRSA infections [5]. Although most MRSA infections in healthcare workers had a mild clinical course, some infections tend to become chronic and can cause severe health problems. This may lead to long-term incapacity, as has

\section{Biomed Central}

(c) 2011 Dulon et al; licensee BioMed Central Ltd. This is an Open Access article distributed under the terms of the Creative Commons Attribution License (http://creativecommons.org/licenses/by/2.0), which permits unrestricted use, distribution, and reproduction in any medium, provided the original work is properly cited. 
been shown by an analysis of the database of a German workers' compensation board [6].

For healthcare facilities, surveillance is an important and generally accepted method to assess the incidence of infection due to multidrug-resistant bacteria and - if necessary to improve infection control measures [7]. Surveillance of MRSA is a means of identifying colonized or infected patients for whom specific control measures may be implemented [8]. Surveillance may be passive, whereby laboratory results from clinical samples are monitored, or active, whereby patients are actively screened for the carrier state in order to identify the entire reservoir. The implementation of a program of active surveillance cultures beside contact precautions is recommended by different national guidelines as a way of preventing nosocomial transmission of MRSA [9-12]. However, it is difficult to determine the range of MRSA rates from existing literature [13], as surveillance is primarily performed during outbreaks and generalization of these results is hampered, as the findings are not applicable to non-outbreak situations [8]. Moreover, as the recognition of MRSA as a hospital problem largely depends on clinical samples or swabs taken either only on admission or selectively for high risk patients, the true case load of a hospital or a specific setting remains largely unknown [14].

In the absence of comprehensive studies on the prevalence of MRSA, rates from MRSA surveillance in hospitals have been transferred to other healthcare settings. However, it has been questioned whether control measures in one setting can be generalized to other settings [9]. This question applies to both epidemic and endemic MRSA and also to specific settings, such as intensive care and non-acute wards, where MRSA may have widely variable transmission dynamics [8].

The aim of this review was to analyze the literature on MRSA prevalence in endemic (non-outbreak) situations in various clinical and long-term care (LTC) settings in European countries. Special emphasis was given to the calculation of MRSA outcomes and to MRSA screening methods.

\section{Methods}

The search was conducted in the PubMed Medline database (2000 through June 2010) and was limited to articles in English or German. Inclusion and exclusion criteria were established on the basis of an exploratory search of the literature.

The search strategy used the keyword 'MRSA' combined with a group of screening-related terms: 'surveillance' OR 'infection control' OR 'prevalence' AND 'healthcare'. Primary inclusion criteria were developed for the initial selection of relevant articles, which were studies presenting MRSA prevalence rates. The initial search yielded 271 citations with an abstract. One of the authors (MD) screened the titles and the abstracts, in order to identify those that met the inclusion criteria, namely the prevalence of colonization or infection with MRSA in patients or residents in clinical and nursing healthcare settings. The exclusion criteria, with the number of excluded abstracts, were as follows: community-associated $(n=64)$, outbreaks $(n=41)$, study region outside Europe $(n=21)$, and healthcare personnel as the sole study population $(\mathrm{n}=$ 6). Another 128 abstracts were excluded as they failed to focus on MRSA prevalence. These included studies evaluating control measures, and those assessing the efficacy of special screening tests, or estimating MRSA transmission. The search was repeated in combination with a group of setting-related terms, namely 'long-term care' OR 'nursing home*' OR 'home care' OR 'mentally handicapped people', resulting in 65 additional abstracts of which 6 were included. Finally, the reference lists of the retrieved articles were checked for additional relevant studies, of which 15 were included. A total of 31 studies were included in the analysis.

Studies were separated into clinical and LTC settings. A data extraction form was developed to collect information from each selected study. This included the following points: 1) Study design: study period, study setting, and study population described by number of eligible participants and actual number of participants; 2) Screening policy: screening procedure and sampling sites; 3) Results: presented in the form of proportions (expressed as the percentage of MRSA cases among patients or among isolates of $S$. aureus) and density rates (expressed as MRSA cases per 1,000 patient days). The extraction of the data was performed by two authors (MD, FH).

The quality of each of the included studies was assessed by using seven criteria, taken from various checklists aimed either at evaluating prevalence surveys [15] or at improving the reporting of observational prevalence studies [16-19]. The criteria were expressed as questions (Table 1). For each study, the answers to these questions were graded as Yes if the question was satisfactorily answered (with a score of one point), No if it was not, and '?' for unclear answers or missing information (with a score of zero points). Two authors (CP, AS) independently assessed the study quality and a third (MD) resolved discrepancies. Study quality was categorized as good ( $>5$ points), fair ( 3 to 5 points) or poor ( $<3$ points). The criteria for rating the study quality in MRSA prevalence studies and the justification for a favorable evaluation will be described briefly.

\section{Outcome definition}

The infection-related outcome had to be clearly defined and described for MRSA colonization and infection [18]. Definition for denominators (e.g. patients, isolates, 
Table 1 Checklist of criteria for assessing the quality of MRSA prevalence surveys

\begin{tabular}{lll}
\hline Number & Name & Content \\
\hline 1 & Outcome definition & Was a valid definition given of the outcome for prevalence, colonization and infection? \\
3 & Time unit & Was the endpoint calculated for a standardized time unit (daily, monthly, yearly)? \\
4 & Target population & Was the target population specified by inclusion or eligibility criteria? \\
5 & Participants & Was the number of included cases reported, e.g. by describing the numbers and reasons for non-participation? \\
6 & Observer bias & Were sources of potential imprecision reported and/or have consequences been discussed? \\
7 & Screening procedure & Were measures described that had been undertaken for standardization of screening measurements? \\
\hline
\end{tabular}

patient days) had to be appropriate [18]: If measurements are performed on the patient's first admission, these are expressed relative to the total number of patients; if measurements are performed for each admission, these are expressed relative to the total number of admissions. If calculations are related to isolates, it should be stated whether only the initial MRSA isolate or all subsequent isolates of a patient during the study period were included. In case of multi-site swabbing, it should be stated whether swabs were cultured separately or as a pooled sample on the same agar plate. For density rates, it should be stated whether denominators were adjusted for total patient days or number of patient-days at risk for new MRSA detection [13].

\section{Time unit}

The outcome had to be calculated for a standardized time unit (daily, weekly, monthly, yearly) [18].

\section{Target population}

The eligibility criteria had to be described [19].

\section{Participants}

The number of cases potentially eligible and actually included had to be reported. A flow chart is recommended for the description of the numbers and reasons for non-participation [19].

\section{Observer bias}

Any efforts to reduce potential sources of bias and imprecision had to be described and the consequences of any potential bias had to be discussed [18].

\section{Screening procedure}

The sampling procedures had to be described [18] and it should be stated if swab specimens were taken by specially trained healthcare workers [20]. Detailed instructions on swab sampling should be given, i.e. use separate swabs for each sampling site of the nares [5], roll the swab three times around in the outer section of each nostril and take the swab from the posterior wall of the throat and not from the mouth [20]. Studies in which the sampling procedure was described in more detail and/or performed by a staff member after appropriate training were therefore scored with one point.

\section{Sampling sites}

The preferred screening method in MRSA prevalence studies is to use swabs. The anterior nares are considered to be the primary colonization site for $S$. aureus $[1,21]$. Screening of the throat in addition to nasal swabs has increased the sensitivity of detection of $S$. aureus among carriers by $20 \%$ to $26 \%$ [20,22]. Although no standards exist for the choice of anatomical sites [4], one scoring point was given when sampling sites included at least the anterior nares and the throat, according to the recommendations by Albrich \& Harbarth [5] and various national guidelines $[12,23,24]$. Studies relying on clinical cultures in addition to screening cultures were only awarded a point when screening cultures included samples from both the anterior nares and the throat. Surveillance studies relying exclusively on clinical cultures (e.g. blood culture of infected patients) were scored with zero points, because clinical cultures taken for therapeutic reasons have shown low sensitivity for detecting the MRSA reservoir [25].

Microbiological media for MRSA screening or use of the polymerase chain reaction were not assessed by the quality score, as no standards exist that define the most effective microbiological media [4].

Extracted data on study design, screening policy and primary MRSA outcomes are described by a narrative approach.

\section{Results}

Table 2 summarizes studies on the prevalence of MRSA in LTC facilities. In 11 studies, surveillance data came from the same setting, nursing homes for the elderly. Two studies (No.8,11/1) included other sectors (rehabilitation center and hospital). The study population was recruited from the residents of the corresponding facility. Study duration varied between 1 and 12 months. In all 11 studies, MRSA prevalence was derived solely from surveillance cultures. Nasal swabs were performed in all studies, though only in six studies by swabbing the anterior nares. In nine studies, screening was performed as multi-site swabbing, most 
Table 2 MRSA prevalence among patients and residents in long-term care facilities

\begin{tabular}{|c|c|c|c|c|c|c|c|c|c|c|c|}
\hline \multirow[t]{3}{*}{ NO. } & \multirow{3}{*}{$\begin{array}{l}\text { Author } \\
\text { (First) } \\
\text { Country, } \\
\text { Year of } \\
\text { Publication }\end{array}$} & \multirow{3}{*}{$\begin{array}{l}\text { Study period, } \\
\text { dates } \\
\text { (months) }\end{array}$} & \multicolumn{2}{|c|}{ Study design } & \multirow{3}{*}{$\begin{array}{l}\text { Study } \\
\text { population } \\
\text { (no. of } \\
\text { residents) (n) }\end{array}$} & \multicolumn{2}{|c|}{ Screening policy } & \multicolumn{3}{|c|}{ Results } & \multirow{3}{*}{$\begin{array}{l}\text { Quality } \\
\text { Rating }^{3}\end{array}$} \\
\hline & & & \multirow{2}{*}{$\begin{array}{l}\text { Study, } \\
\text { setting } \\
\text { (no. of } \\
\text { units) (n) }\end{array}$} & \multirow{2}{*}{$\begin{array}{l}\text { Basic number of } \\
\text { cases } \\
\text { potentially } \\
\text { eligible }(n)\end{array}$} & & \multirow{2}{*}{$\begin{array}{l}\text { Screening } \\
\text { methods }\end{array}$} & \multirow{2}{*}{$\begin{array}{l}\text { Swabbing } \\
\text { sites }\end{array}$} & \multirow{2}{*}{$\begin{array}{l}\text { Number of MRSA } \\
\text { patients colonised } \\
\text { (C) or infected (I) (n) }\end{array}$} & \multicolumn{2}{|c|}{ Proportion of MRSA } & \\
\hline & & & & & & & & & $\begin{array}{l}\% \text { MRSA among } \\
\text { patients Mean } \\
(95 \% \text { CI) })^{1} \text { Range }^{2}\end{array}$ & $\begin{array}{l}\% \text { MRSA among } \\
\text { S.aureus isolates } \\
\text { Mean }(95 \% \mathrm{Cl})^{1}\end{array}$ & \\
\hline 1 & $\begin{array}{l}\text { Baldwin } \\
\text { [36] } \\
\text { Northern } \\
\text { Ireland } \\
2009\end{array}$ & 2005-2006 (9) & $\mathrm{NH}-\mathrm{E}(45)$ & 1.678 & $1.111^{4}$ & $\begin{array}{l}\text { Non-selective } \\
\text { on one day in } \\
\text { each unit }\end{array}$ & $\begin{array}{l}\text { Nares, urine, } \\
\text { wounds, } \\
\text { inv.devices }\end{array}$ & 267 (C) & $\begin{array}{l}23.3(18.8-27.7) \\
\text { Range, } 0 \text { to } 73\end{array}$ & & $\begin{array}{c}\text { Good } \\
1 Y ; 2 Y ; 3 Y ; 4 Y ; \\
5 Y ; 6 Y ; 7 N\end{array}$ \\
\hline 2 & $\begin{array}{l}\text { Barr } \\
{[38]} \\
\text { UK } \\
2007\end{array}$ & 2005 (2) & $\begin{array}{l}\text { Care homes } \\
\text { for the } \\
\text { elderly (39) }\end{array}$ & 1.342 & $715^{4}$ & Non-selective & Nose & 159 (C) & $\begin{array}{l}22.0(18.0-27.0) \\
\text { Range, } 0 \text { to } 50\end{array}$ & 54.0 & $\begin{array}{c}\text { Fair } \\
1 \mathrm{~N} ; 2 \mathrm{~N} ; 3 \mathrm{Y} ; 4 \mathrm{Y} ; \\
5 \mathrm{Y} ; 6 \mathrm{6} ; 7 \mathrm{~N}\end{array}$ \\
\hline 3 & $\begin{array}{l}\text { Baum, von } \\
\text { [37] } \\
\text { Germany } \\
2002\end{array}$ & $1999-2000(12)$ & NH-E (47) & 3.864 & $3.236^{4}$ & Non-selective & $\begin{array}{l}\text { Nares, skin } \\
\text { defects }\end{array}$ & $36(C)$ & $\begin{array}{l}1.1(0.75-1.47) \\
\text { Range, } 0 \text { to } 18.2\end{array}$ & & $\begin{array}{c}\text { Fair } \\
1 \mathrm{~N} ; 2 \mathrm{Y} ; 3 \mathrm{3Y} ; 4 \mathrm{Y} ; \\
5 \mathrm{Y} ; 6 \mathrm{~N} ; 7 \mathrm{~N}\end{array}$ \\
\hline 4 & $\begin{array}{l}\text { Brugnaro } \\
{[39]} \\
\text { Italy } \\
2009\end{array}$ & $2006(1)$ & $\begin{array}{l}\text { NH-E (2 } \\
\text { with } 15 \\
\text { units) }\end{array}$ & 570 & $551^{4}$ & $\begin{array}{l}\text { Non-selective } \\
\text { on a single day } \\
\text { in each unit }\end{array}$ & Nares & $43(\mathrm{C})$ & $\begin{array}{l}7.8(5.7-10.4) \\
\text { Range, } 0 \text { to } 18\end{array}$ & & $\begin{array}{c}\text { Fair } \\
1 \mathrm{~N} ; 2 \mathrm{2Y} ; 3 \mathrm{Y} ; 4 \mathrm{Y} ; \\
5 \mathrm{Y} ; 6 \mathrm{~N} ; 7 \mathrm{~N}\end{array}$ \\
\hline 5 & $\begin{array}{l}\text { Cretnik } \\
{[40]} \\
\text { Slovenia } \\
2005\end{array}$ & $2001(1)$ & $\mathrm{NH}-\mathrm{E}(1)$ & 127 & 107 & Non-selective & $\begin{array}{l}\text { Nares, skin } \\
\text { lesions }\end{array}$ & $10(C)$ & 9.3 & & $\begin{array}{c}\text { Poor } \\
1 \mathrm{~N} ; 2 \mathrm{~N} ; 3 \mathrm{Y} ; 4 \mathrm{Y} ; \\
5 \mathrm{~N} ; 6 \text { ? } 7 \mathrm{~N}\end{array}$ \\
\hline 6 & $\begin{array}{l}\text { Denis } \\
\text { [33] } \\
\text { Belgium } \\
2009\end{array}$ & 2005 (9) & $\begin{array}{l}\text { A random } \\
\text { sample of } \\
\mathrm{NH}-\mathrm{E}(60)\end{array}$ & NA & 2.953 & $\begin{array}{l}\text { Non-selective } \\
\text { on the same } \\
\text { day in each unit }\end{array}$ & $\begin{array}{l}\text { Nares, } \\
\text { throat, } \\
\text { wounds, } \\
\text { cath. urine }\end{array}$ & $587(C)$ & $\begin{array}{l}19.5(16.4-21.5) \\
\text { Range, } 2 \text { to } 42.9\end{array}$ & 39.1 & $\begin{array}{c}\text { Fair } \\
1 \mathrm{~N} ; 2 \mathrm{Y} ; 3 \mathrm{3Y} ; 4 \mathrm{Y} ; \\
5 \mathrm{Y} ; 6 \mathrm{~N} ; 7 \mathrm{Y}\end{array}$ \\
\hline 7 & $\begin{array}{l}\text { Heuck } \\
{[41]} \\
\text { Germany } \\
2000\end{array}$ & NA & $\mathrm{NH}-\mathrm{E}(31)$ & $?$ & $1.342^{4}$ & Non-selective & $\begin{array}{l}\text { Nares, } \\
\text { throat, } \\
\text { wounds }\end{array}$ & $32(\mathrm{C})$ & $\begin{array}{l}2.4 \\
\text { Range, } 0 \text { to } 2.9\end{array}$ & 6.0 & $\begin{array}{c}\text { Poor } \\
1 \mathrm{~N} ; 2 \mathrm{~N} ; 3 \mathrm{Y} ; 4 \mathrm{~N} ; \\
5 \mathrm{~N} ; 6 \mathrm{~N} ; 7 \mathrm{Y}\end{array}$ \\
\hline 8 & $\begin{array}{l}\text { Heudorf } \\
{[42]} \\
\text { Germany } \\
2001\end{array}$ & 1999-2000 NA & $\begin{array}{l}\mathrm{NH}-\mathrm{E}(7) ; \\
\text { geriatric } \mathrm{RC} \\
\text { (1) }\end{array}$ & $?$ & $\begin{array}{l}\mathrm{NH}-\mathrm{E}: 359 \text { RC: } \\
42^{4}\end{array}$ & Non-selective & Nose, throat & NH-E: 8 (C) RC: 2 (C) & NH-E: 2.2 RC: 4.8 & & $\begin{array}{c}\text { Fair } \\
1 \mathrm{~N} ; 2 \mathrm{~N} ; 3 \mathrm{Y} ; 4 \mathrm{Y} ; \\
5 \mathrm{Y} ; 6 \mathrm{~N} ; 7 \mathrm{Y}\end{array}$ \\
\hline 9 & $\begin{array}{l}\text { Hoefnagels- } \\
\text { Schuermans } \\
\text { [43] } \\
\text { Belgium }\end{array}$ & 1997 (3) & $\mathrm{NH}-\mathrm{E}(17)$ & $?$ & 2.857 & $\begin{array}{l}\text { Non-selective; } \\
\text { one day } \\
\text { sampling in } \\
\text { each unit }\end{array}$ & $\begin{array}{l}\text { Nose, } \\
\text { perineum }\end{array}$ & $141(C)$ & $4.9(4.38-6.09)$ & 19.1 & $\begin{array}{c}\text { Poor } \\
1 \mathrm{~N} ; 2 \mathrm{Y} ; 3 \mathrm{~N} ; 4 \mathrm{~N} ; \\
5 \mathrm{~N} ; 6 \mathrm{Y} ; 7 \mathrm{~N}\end{array}$ \\
\hline
\end{tabular}


Table 2 MRSA prevalence among patients and residents in long-term care facilities (Continued)

\begin{tabular}{|c|c|c|c|c|c|c|c|c|c|c|c|}
\hline 10 & $\begin{array}{l}\text { Neuhaus } \\
{[44]} \\
\text { Germany } \\
2002\end{array}$ & 2000-2001 (12) & $\mathrm{NH}-\mathrm{E}(61)$ & $?$ & $1.057^{4}$ & Non-selective & $\begin{array}{l}\text { Nose, } \\
\text { throat, } \\
\text { wounds }\end{array}$ & 32 (C) 3 (I) & $3.0(2.1-4.2)$ & $6.3(4.3-8.8)$ & $\begin{array}{c}\text { Fair } \\
1 Y ; 2 N ; 3 Y ; 4 N ; \\
5 N ; 6 N ; 7 Y\end{array}$ \\
\hline $\begin{array}{l}11 / \\
1\end{array}$ & $\begin{array}{l}\text { Woltering } \\
{[45]} \\
\text { Germany } \\
2008\end{array}$ & NA (4) & NH-E (5) & 441 & $265^{4}$ & $\begin{array}{l}\text { Non-selective } \\
\text { on a 2-days } \\
\text { period in each } \\
\text { unit }\end{array}$ & $\begin{array}{l}\text { Nose, } \\
\text { throat, } \\
\text { wounds }\end{array}$ & $4(C)$ & $2.3(0.8-4.9)$ & 5.9 & $\begin{array}{c}\text { Good } \\
1 \mathrm{~N} ; 2 \mathrm{2Y} 3 \mathrm{YY} ; 4 \mathrm{4Y} ; \\
5 \mathrm{SY} ; 6 \mathrm{~N} ; 7 \mathrm{Y}\end{array}$ \\
\hline
\end{tabular}

1 : Pooled mean calculated as a combined prevalence rate over all units with $95 \% \mathrm{Cl}$.

2 : Range within the individual units.

3: Levels for study quality: Good (= 6 and 7 points), fair ( $=3-5$ points), poor (= 1 and 2 points). Numbers belong to the questions as illustrated in Table 1.

4: Participants who were able to give informed consent or informed consent were given by their relatives. Abbreviations: Cl, Confidence intervals; $\mathrm{N}$, no; $\mathrm{NH}$-(E), Nursing home for the elderly; RC, Rehabilitation centre;

Y, Yes; NA, No Answer; ?, unclear or missing information. 
often by additional swabs taken from skin lesions or wounds. Swabbing of the throat was performed routinely in five studies. The screening was non-selective in all studies with regard to study population. The screening procedure was described in more detail in five studies (on a single day or on a two days period in each unit). The quality level was assigned as 'good' in two studies, as 'fair' in six studies, and as 'poor' in three studies. Prevalence in LTC facilities varied from $1 \%$ to $23 \%$, if the percentage of MRSA among residents is taken, and from 5\% to $54 \%$, if the percentage of MRSA among S. aureus isolates is taken. MRSA proportions of around $20 \%$ or higher were found in UK, Northern Ireland and Belgium.

Table 3 summarizes studies on the prevalence of MRSA in clinical settings. A total of 20 studies were included, of which one study presented two surveillance approaches (No.19). Another study was considered, which included both clinical and LTC facilities (No.11/ 2 ). Four studies were designed as multi-center surveillance studies (No.14,20,23,29). Study setting included different forms of hospitals (university, primary or tertiary care), specialized units (intensive care (ICU), surgery, neurology, vascular, emergency, orthopedics), rehabilitation centers and one laboratory facility. Study duration varied across a range from less than 1 month to 72 months, with accumulation round 4 months. Four groups of study population were found: admissions (in general or as specified admissions to ICU, to emergency, or to geriatrics); hospitalized patients (more than 24 or 48 hours in hospital); special groups of patients (of greater age, at discharge to home care, being a trauma patient); and S. aureus isolates of infected patients. Identification of MRSA cases was achieved in 15 studies by swabs for surveillance purposes exclusively, in three studies additionally by clinical cultures, and in another three studies exclusively via clinical cultures obtained from infected patients for therapeutic reasons.

Screening was performed at different time points: on admission within 24 to 48 hours or during hospital stay (one week after admission, on the $21^{\text {st }}$ day of admission, within three days before discharge to home care, or by a weekly screening cycle). Nasal swabbing was performed in 18 studies, but anterior nares were used in five studies only. Wounds were the second most frequently swabbed screening site (11 studies). Additional anatomical sites were either the throat, groin, axillae, or perineum. Considering the seven quality criteria, the quality level was assigned as 'good' in two studies (plus one already counted in Table 2; No.11), as 'fair' in 14 studies, and as 'poor' in four studies.

The prevalence rates in clinical settings varied from $0.1 \%$ to $24.0 \%$ if the percentage of MRSA among patients is taken and from $5.5 \%$ to $29.7 \%$ if the percentage of MRSA among $S$. aureus isolates is taken. In surveillance studies analyzing solely clinical samples of infected patients, the percentage of MRSA among $S$. aureus isolates varied between $13.8 \%$ and $21.5 \%$ (No.20,23,29). Prevalence density was calculated in four studies and varied between 0.4 and 0.7 MRSA-positive cases per 1,000 patient days, when all patients gave rise to the denominator (No.13,14,19), and was 4 MRSA isolates per 1,000 patient days when calculated on the basis of clinical samples of infected patients (No.23).

With regard to screening procedures, the MRSA percentage varied from less than $1 \%$ to $24 \%$ when swabs were taken within 24 to 48 hours of admission, from $3.4 \%$ to $15.8 \%$ when swabs were taken during the hospital stay, and from less than $1 \%$ to $7.9 \%$ when swabs were taken on admission of patients with a high risk for MRSA carrier status. With regard to comparable clinical settings, the MRSA percentage among patients varied from $0.48 \%$ to $11.5 \%$ in intensive care units $(n=3)$, from $7.9 \%$ to $14.6 \%$ in acute (geriatric) care wards ( $\mathrm{n}=$ 2 ), from $1.6 \%$ to $24.6 \%$ in surgeries (orthopedic, trauma, or vascular) $(n=5)$, from less than $1 \%$ to $15.8 \%$ for different wards in hospitals in general ( $n=10)$, and from $1.2 \%$ to $2.1 \%$ in rehabilitation centers $(n=2)$.

\section{Discussion}

In this review, 31 studies on MRSA prevalence rates in endemic situations in different healthcare settings in eight European countries were analyzed. Prevalence rates, defined as the proportion of MRSA-positive patients, varied widely over a range between less than $1 \%$ and $24 \%$. The variations in MRSA proportion were less marked between acute-care and long-term care setting, but rather between single wards in each setting. Study quality was assessed as good in only four studies. Most studies $(n=20)$ were assessed as being of fair quality and seven as of poor quality. The aim of this review was to present prevalence rates for endemic MRSA in all kinds of healthcare sectors, but was not fully achieved. In fact, MRSA rates are presented for a few different clinical sectors and not for a variety of LTC sectors, as in the LTC setting, surveillance was performed only in nursing homes for the elderly.

In the last 10 years, significant increases in MRSA (expressed as percentage of $S$. aureus blood isolates) have been shown in European countries [3]. Time trends for resistance proportions for MRSA have been presented by two other surveillance systems, with an increase of MRSA from 1\% in 1990 to $20 \%$ in 2007 [26], and stable proportions between $20 \%$ and $26 \%$ in the years between 2001 and 2007 [27]. The MRSA rates of the studies analyzed in this review show a different distribution, with the most frequent rates being less than $10 \%$ (expressed as MRSA among patients). The range of MRSA rates may reflect differences in specific national 
Table 3 MRSA prevalence among patients in clinical settings

\begin{tabular}{|c|c|c|c|c|c|c|c|c|c|c|c|c|c|}
\hline \multirow[t]{3}{*}{ NO. } & \multirow{3}{*}{$\begin{array}{l}\text { Author, } \\
\text { First } \\
\text { Country, } \\
\text { Year of } \\
\text { Publication }\end{array}$} & \multirow{3}{*}{$\begin{array}{l}\text { Study } \\
\text { period } \\
\text { (no. of } \\
\text { months) }\end{array}$} & \multicolumn{3}{|c|}{ Study design } & \multicolumn{2}{|c|}{ Screening policy } & \multicolumn{4}{|c|}{ Results } & \multirow[t]{3}{*}{ Comments } & \multirow[t]{3}{*}{$\begin{array}{l}\text { Quality } \\
\text { rating }\end{array}$} \\
\hline & & & Study & Study & Basic no. of & Screening & Swabbing & No. of MRSA & \multirow[b]{2}{*}{$\begin{array}{l}\% \text { MRSA } \\
\text { among } \\
\text { patients } \\
\text { Mean }{ }^{2} \\
(95 \% \mathrm{Cl}) \\
\text { Range }^{3}\end{array}$} & \multirow[b]{2}{*}{$\begin{array}{l}\% \text { MRSA } \\
\text { among S. } \\
\text { aureus } \\
\text { isolates } \\
\text { Mean }^{2}\end{array}$} & \multirow[b]{2}{*}{$\begin{array}{l}\text { Density (no. } \\
\text { of MRSA } \\
\text { per } 1,000 \\
\text { patients } \\
\text { days }\end{array}$} & & \\
\hline & & & & & & & & & & & & & \\
\hline 12 & $\begin{array}{l}\text { Chaberny } \\
{[46]} \\
\text { Germany } \\
2008\end{array}$ & $\begin{array}{l}2005 \\
(<1)\end{array}$ & $\begin{array}{l}\text { ICU (1), } \\
\text { surgery (1), } \\
\text { neurology (1), } \\
\text { internal } \\
\text { medicine (1) }\end{array}$ & Patients & 700/509 & $\begin{array}{l}\text { On a given } \\
\text { study day }\end{array}$ & $\begin{array}{l}\text { Nares, throat, } \\
\text { skin lesions }\end{array}$ & 27 (C) & $\begin{array}{l}.3(3.5-7.7) \\
\text { ICU: } 11.5^{\mathrm{a}} \\
\text { Surgery: } 5.5^{\mathrm{a}} \\
\text { Neuro. } 11.8 \\
\text { Int. med.: } 5.1\end{array}$ & 19.0 & & $\begin{array}{l}{ }^{a} \text { Units with } \\
\text { established } \\
\text { admission } \\
\text { screening }\end{array}$ & $\begin{array}{l}\text { Good } \\
1 Y ; 2 Y ; \\
3 Y ; 4 Y ; \\
5 Y ; 6 Y ; \\
7 Y\end{array}$ \\
\hline 13 & $\begin{array}{l}\text { Chaberny } \\
\text { [47] } \\
\text { Germany } \\
2005\end{array}$ & $\begin{array}{l}2002 \\
(12)\end{array}$ & ICU (4) & Admissions & $? / 188.615$ & $\begin{array}{l}\text { On } \\
\text { admission } \\
\text { of patients } \\
\text { at risk for } \\
\text { MRSA } \\
\text { Clinical } \\
\text { samples }^{5}\end{array}$ & n.r. & 505 (C) 404 (I) & 0.48 & & $\begin{array}{l}0.64^{\mathrm{a}} \\
0.29^{\mathrm{a}, \mathrm{b}}\end{array}$ & $\begin{array}{l}\text { a } \\
\text { Admissions } \\
\text { b Noso- } \\
\text { comial }\end{array}$ & $\begin{array}{l}\text { Fair } \\
1 Y ; 2 Y ; \\
3 N ; 4 Y ; \\
5 N ; 6 Y ; \\
7 ?\end{array}$ \\
\hline 14 & $\begin{array}{l}\text { Chaberny } \\
\text { [7] } \\
\text { Germany } \\
2007\end{array}$ & $\begin{array}{l}2004 \\
(12)\end{array}$ & Hospital (31) & $\begin{array}{l}\text { Hospita-lized } \\
\text { patients }\end{array}$ & $? / 660.042$ & $\begin{array}{l}\text { During } \\
\text { hospital } \\
\text { stay } \\
\text { Clinical } \\
\text { samples }^{5}\end{array}$ & Nares & 2.786 (C) 1.429 (I) & & & $\begin{array}{l}0.71^{a} \\
0.27^{a, b}\end{array}$ & $\begin{array}{l}\text { a } \\
\text { Admissions } \\
\text { b Noso- } \\
\text { comial }\end{array}$ & $\begin{array}{l}\text { Fair } \\
1 Y ; 2 Y ; \\
3 Y ; 4 N ; \\
5 Y ; 6 N ; \\
7 N\end{array}$ \\
\hline 15 & $\begin{array}{l}\text { Eveillard } \\
\text { [48] } \\
\text { France } \\
2002\end{array}$ & $2000(1)$ & $\begin{array}{l}\text { Acute geriatric } \\
\text { ward (2) }\end{array}$ & Patients & $244 / 239$ & $\begin{array}{l}\text { On the first } \\
\text { day of } \\
\text { admission }\end{array}$ & $\begin{array}{l}\text { Nares, } \\
\text { wounds }\end{array}$ & $35(C)$ & $\begin{array}{l}14.6(10.1- \\
19.1)\end{array}$ & & & & $\begin{array}{l}\text { Fair } \\
1 \mathrm{~N} ; 2 \mathrm{Y} ; \\
3 \mathrm{Y} ; 4 \mathrm{Y} ; \\
5 \mathrm{~N} ; 6 \mathrm{~N} ; \\
7 \mathrm{~N}\end{array}$ \\
\hline 16 & $\begin{array}{l}\text { Anonymous } \\
{[49]} \\
\text { Germany } \\
2010\end{array}$ & 2008 (1) & $\mathrm{RC}(6), \mathrm{GH}(8)$ & Patients & $? / 6.985$ & $\begin{array}{l}\text { On } \\
\text { admission } \\
\text { during } \\
\text { initial exa- } \\
\text { mination }\end{array}$ & $\begin{array}{l}\text { Nose, } \\
\text { throat }\end{array}$ & $95(\mathrm{C})$ & $\begin{array}{l}\mathrm{RC}: 2.1 \\
\mathrm{GH}: 1.2\end{array}$ & $\begin{array}{l}\text { RC: } 9.4 \\
\text { GH: } 5.5\end{array}$ & & & $\begin{array}{l}\text { Fair } \\
1 \mathrm{~N} ; 2 \mathrm{Y} ; \\
3 \mathrm{Y} ; 4 \mathrm{~N} ; \\
5 \mathrm{~N} ; 6 ? ; \\
7 \mathrm{Y}\end{array}$ \\
\hline 17 & $\begin{array}{l}\text { Hassan } \\
\text { [50] } \\
\text { Ireland } \\
2008\end{array}$ & 2005 (3) & $\begin{array}{l}\text { Orthopedic } \\
\text { ward (2) }\end{array}$ & Patients & $690 / 686$ & $\begin{array}{l}\text { Within } 24 \\
\text { hrs of } \\
\text { admission }\end{array}$ & $\begin{array}{l}\text { Nose, } \\
\text { perineum, } \\
\text { surgical } \\
\text { wounds }\end{array}$ & $27(C)$ & 3.9 & & & & $\begin{array}{l}\text { Fair } \\
1 \mathrm{~N} ; 2 \mathrm{~N} ; \\
3 \mathrm{Y} ; 4 \mathrm{Y} ; \\
5 \mathrm{~N} ; 6 \mathrm{Y} ; \\
7 \mathrm{~N}\end{array}$ \\
\hline 18 & $\begin{array}{l}\text { Hori } \\
{[14]} \\
\text { UK } \\
2002\end{array}$ & $2000(4)$ & UH (1) & $\begin{array}{l}\text { Patients older } \\
\text { than } 64 \text { years }\end{array}$ & $431 / 342$ & $\begin{array}{l}\text { On the } \\
21^{\text {st }} \text { day } \\
\text { after } \\
\text { admission }\end{array}$ & Nares & $54(C)$ & 15.8 & & & & $\begin{array}{l}\text { Fair } \\
1 N ; 2 Y ; \\
3 Y ; 4 Y ; \\
5 Y ; 6 N ; \\
7 N\end{array}$ \\
\hline
\end{tabular}


Table 3 MRSA prevalence among patients in clinical settings (Continued)

\begin{tabular}{|c|c|c|c|c|c|c|c|c|c|c|c|c|c|}
\hline $\begin{array}{l}19 / \\
1\end{array}$ & $\begin{array}{l}\text { Kappstein } \\
{[51]} \\
\text { Germany } \\
2009\end{array}$ & $\begin{array}{l}2000- \\
2005 \\
(72)\end{array}$ & UH (1) & Patients & $?$ & $\begin{array}{l}\text { On } \\
\text { admission } \\
\text { Clinical } \\
\text { samples }^{5}\end{array}$ & Nose & 489 (C) 38 (I) & & & 0.42 & & $\begin{array}{l}\text { Poor } \\
1 \mathrm{Y} ; 2 \mathrm{~N} ; \\
3 \mathrm{~N} ; 4 \mathrm{~N} ; \\
5 \mathrm{~N} ; 6 \mathrm{~N} ; \\
7 \mathrm{~N}\end{array}$ \\
\hline $\begin{array}{l}19 / \\
2\end{array}$ & $\begin{array}{l}\text { Kappstein } \\
{[51]} \\
\text { Germany } \\
2009\end{array}$ & $\begin{array}{l}2002- \\
2005 \\
(42)\end{array}$ & UH (1) & Patients & $141.249 / 29.692$ & $\begin{array}{l}\text { Within } \\
48 \text { hrs of } \\
\text { admission }\end{array}$ & $\begin{array}{l}\text { Nose, } \\
\text { wounds }\end{array}$ & $231(C)$ & 0.78 & & & & \\
\hline 20 & $\begin{array}{l}\text { Kresken } \\
{[26]} \\
\text { Germany } \\
2009\end{array}$ & $\begin{array}{l}2007 \\
(1)\end{array}$ & LF (26) & $\begin{array}{l}\text { S. aureus } \\
\text { isolates }\end{array}$ & $? / 872$ & $\begin{array}{l}\text { Clinical } \\
\text { samples }^{5}\end{array}$ & & 159 isolates & & 20.3 & & & $\begin{array}{l}\text { Poor } \\
1 N ; 2 Y ; \\
3 Y ; 4 N ; \\
5 N ; 6 N ; \\
7 N\end{array}$ \\
\hline 21 & $\begin{array}{l}\text { Lucet } \\
\text { [52] } \\
\text { France } \\
2009\end{array}$ & $\begin{array}{l}2003- \\
2004 \\
(14)\end{array}$ & $\begin{array}{l}\text { Hospitals for } \\
\text { primary and } \\
\text { tertiary care } \\
\text { (16) }\end{array}$ & $\begin{array}{l}\text { Patients at } \\
\text { discharge to } \\
\text { home care, > } \\
48 \text { hrs in } \\
\text { hospital }\end{array}$ & $2.025 / 1.501$ & $\begin{array}{l}\text { Within } \\
3 \text { days } \\
\text { before } \\
\text { discharge }\end{array}$ & $\begin{array}{l}\text { Nose, } \\
\text { chronic skin } \\
\text { lesions }\end{array}$ & $191(C)$ & $\begin{array}{l}12.7 \\
(11.0-14.5)\end{array}$ & & & $\begin{array}{l}\text { a Obstetric } \\
\text { patients } \\
\text { excluded }\end{array}$ & $\begin{array}{l}\text { Fair } \\
1 Y ; 2 N ; \\
3 Y ; 4 Y ; \\
5 Y ; 6 Y ; \\
7 N\end{array}$ \\
\hline 22 & $\begin{array}{l}\text { Lucet } \\
\text { [53] } \\
\text { France } \\
2005\end{array}$ & $\begin{array}{l}2002 \\
(3,5)\end{array}$ & $\begin{array}{l}\text { Acute care } \\
\text { ward in a } \\
\text { hospital (1) }\end{array}$ & $\begin{array}{l}\text { Patients older } \\
\text { than } 75 \text { years, } \\
>24 \text { hrs in } \\
\text { hospital }\end{array}$ & $1.434 / 797$ & $\begin{array}{l}\text { Within } 48 \\
\text { hrs of } \\
\text { admission }\end{array}$ & $\begin{array}{l}\text { Nose, } \\
\text { skin breaks }\end{array}$ & $63(C)$ & $\begin{array}{l}7.9^{\mathrm{a}} \\
(6.0-9.8)\end{array}$ & $29.7^{\mathrm{a}}$ & & a & $\begin{array}{l}\text { Fair } \\
1 Y ; 2 N ; \\
3 Y ; 4 Y ; \\
5 Y ; 6 Y ; \\
7 N\end{array}$ \\
\hline 23 & $\begin{array}{l}\text { Meyer } \\
{[54]} \\
\text { Germany } \\
2006\end{array}$ & $\begin{array}{l}2001- \\
2004 \\
(48)\end{array}$ & ICU (40) & $\begin{array}{l}\text { S. aureus } \\
\text { isolates of } \\
\text { patients with } \\
\text { noso-comial } \\
\text { infections }\end{array}$ & $? / 12.238$ & $\begin{array}{l}\text { Clinical } \\
\text { samples }^{5}\end{array}$ & & 2.631 isolates & & 21.5 & 4.4 & & $\begin{array}{l}\text { Good } \\
1 Y ; 2 Y ; \\
3 Y ; 4 Y ; \\
Y 5 ; 6 N ; \\
7 N\end{array}$ \\
\hline 24 & $\begin{array}{l}\text { Morange- } \\
\text { Saussier } \\
{[55]} \\
\text { France } \\
2006\end{array}$ & $\begin{array}{l}2004 \\
(4)\end{array}$ & $\begin{array}{l}\text { Vascular } \\
\text { surgery (1) }\end{array}$ & $\begin{array}{l}\text { Patients, } \\
>24 \text { hrs in } \\
\text { hospital }\end{array}$ & $? / 308$ & $\begin{array}{l}\text { On } \\
\text { admission } \\
\text { and } 1 \text { wk } \\
\text { thereafter }\end{array}$ & Nares & $13(\mathrm{C})$ & 4.2 & 27.0 & & & $\begin{array}{l}\text { Fair } \\
1 Y ; 2 Y ; \\
3 Y ; 4 Y ; \\
5 N ; 6 N ; \\
7 N\end{array}$ \\
\hline 25 & $\begin{array}{l}\text { Gopal Rao } \\
{[29]} \\
\text { UK } \\
2007\end{array}$ & $\begin{array}{l}2004- \\
2005 \\
(12)\end{array}$ & $\begin{array}{l}\text { GH (1); } \\
\text { emergency } \\
\text { department }\end{array}$ & $\begin{array}{l}\text { Adult } \\
\text { emergency } \\
\text { admissions }\end{array}$ & 13.826/7.801 & $\begin{array}{l}\text { Prior to } \\
\text { admission }\end{array}$ & $\begin{array}{l}\text { Nose, } \\
\text { axillae }\end{array}$ & $\begin{array}{l}670(C) \\
433(C)\end{array}$ & $\begin{array}{l}8.6^{\mathrm{a}} \\
6.7^{\mathrm{b}}\end{array}$ & & & $\begin{array}{l}\text { a } \\
\text { Admissions } \\
\text { b Patients }\end{array}$ & $\begin{array}{l}\text { Fair } \\
1 \mathrm{~N} ; 2 \mathrm{2Y} ; \\
3 \mathrm{Y} ; 4 \mathrm{Y} ; \\
5 \mathrm{Y} ; 6 \mathrm{~N} ; \\
7 \mathrm{~N}\end{array}$ \\
\hline 26 & $\begin{array}{l}\text { Reilly } \\
{[56]} \\
\text { UK } \\
2010\end{array}$ & $\begin{array}{l}2008 \\
(5)\end{array}$ & GH (6) & $\begin{array}{l}\text { Emergency } \\
(68 \%) \text { and } \\
\text { elective (32\%) } \\
\text { admissions }\end{array}$ & $29.690 / 26.160$ & $\begin{array}{l}\text { On } \\
\text { admission } \\
\text { or at pre- } \\
\text { admission } \\
(7.5 \%)\end{array}$ & $\begin{array}{l}\text { Nose, } \\
\text { wounds, } \\
\text { invasive } \\
\text { device sites }\end{array}$ & $988(C+1)$ & $\begin{array}{l}3.8^{\mathrm{a}} \\
(3.5-4.0)\end{array}$ & & & a & $\begin{array}{l}\text { Fair } \\
1 N ; 2 N ; \\
3 Y ; 4 Y ; \\
5 Y ; 6 Y ; \\
7 N\end{array}$ \\
\hline 27 & $\begin{array}{l}\text { Tai } \\
{[57]} \\
\text { UK } \\
2004\end{array}$ & $\begin{array}{l}2000 \\
(12)\end{array}$ & $\begin{array}{l}\text { Orthopedic } \\
\text { and trauma } \\
\text { surgery (1) }\end{array}$ & $\begin{array}{l}\text { Patients } \\
\text { at high risk for } \\
\text { MRSA }\end{array}$ & $1.879 / 121$ & $\begin{array}{l}\text { On } \\
\text { admission } \\
\text { Clinical } \\
\text { samples }^{5}\end{array}$ & $\begin{array}{l}\text { Nose, throat, } \\
\text { axillae, } \\
\text { groins, } \\
\text { wounds }\end{array}$ & $\begin{array}{l}10(\mathrm{C}) \\
21(\mathrm{I})\end{array}$ & 1.6 & & & & $\begin{array}{l}\text { Poor } \\
1 \mathrm{Y} ; 2 \mathrm{~N} ; \\
3 \mathrm{Y} ; 4 \mathrm{~N} ; \\
5 \mathrm{~N} ; 6 \mathrm{~N} ; \\
7 \mathrm{~N}\end{array}$ \\
\hline
\end{tabular}


Table 3 MRSA prevalence among patients in clinical settings (Continued)

\begin{tabular}{|c|c|c|c|c|c|c|c|c|c|c|c|c|c|}
\hline 28 & $\begin{array}{l}\text { Thompson } \\
{[58]} \\
\text { UK } \\
2004\end{array}$ & $\begin{array}{l}2001- \\
2004 \\
(30)\end{array}$ & ICU (1) & $\begin{array}{l}\text { Admissions to } \\
\mathrm{ICU}\end{array}$ & $1.472 / 1.361$ & $\begin{array}{l}\text { On } \\
\text { admission } \\
\text { Weekly } \\
\text { screening } \\
\text { cycle } \\
\text { Clinical } \\
\text { samples }^{5}\end{array}$ & Nose, groins & $\begin{array}{l}119(C+1) \\
68(C+1)^{b}\end{array}$ & $\begin{array}{l}8.7^{\mathrm{a}}(6.1- \\
10.2)\end{array}$ & & & $\begin{array}{l}\text { a } \\
\text { Admissions } \\
\text { b } \\
\text { Nosocomial } \\
\text { Incidence: } \\
1^{\text {st }} w k \text { : } \\
7.5 \% ; 2^{\text {nd }} \text { to } \\
4^{\text {th }} w k: \\
20.3 \%\end{array}$ & $\begin{array}{l}\text { Fair } \\
1 Y ; 2 ? ; \\
3 Y ; 4 ? ; \\
5 Y ; 6 N ; \\
7 \mathrm{~N}\end{array}$ \\
\hline 29 & $\begin{array}{l}\text { Tiemersma } \\
{[3]} \\
\text { Germany } \\
2004\end{array}$ & $\begin{array}{l}1999- \\
2002 \\
(36)\end{array}$ & Hospitals (25) & $\begin{array}{l}\text { S. aureus } \\
\text { blood isolates }\end{array}$ & $? / 3.757$ & $\begin{array}{l}\text { Blood } \\
\text { cultures }^{5}\end{array}$ & & 600 isolates & & 13.8 & & & $\begin{array}{l}\text { Poor } \\
1 Y ; 2 ? ; \\
3 N ; 4 Y ; \\
5 N ; 6 N ; \\
7 N\end{array}$ \\
\hline 30 & $\begin{array}{l}\text { Vos } \\
{[59]} \\
\text { NL } \\
2009\end{array}$ & $\begin{array}{l}2000- \\
2004 \\
(60)\end{array}$ & $\mathrm{UH}(1)$ & $\begin{array}{l}\text { Admissions } \\
\text { at high risk for } \\
\text { MRSA }\end{array}$ & $\begin{array}{l}\text { ?/ } \\
21.598\end{array}$ & $\begin{array}{l}\text { On } \\
\text { admission }\end{array}$ & $\begin{array}{l}\text { Nose, throat, } \\
\text { perineum, } \\
\text { invasive } \\
\text { devices, } \\
\text { wounds }\end{array}$ & $123(\mathrm{C})$ & 0.10 & & $0.0028^{a}$ & $\begin{array}{l}\text { a Related to } \\
\text { bacteremia } \\
\text { cases }\end{array}$ & $\begin{array}{l}\text { Fair } \\
1 \mathrm{~N} ; 2 \mathrm{Y} ; \\
3 \mathrm{Y} ; 4 \mathrm{~N} ; \\
5 \mathrm{Y} ; 6 \mathrm{~N} ; \\
7 \mathrm{Y} ;\end{array}$ \\
\hline 31 & $\begin{array}{l}\text { Walley } \\
{[60]} \\
\text { UK } \\
2009\end{array}$ & $\begin{array}{l}2003 \\
(3)\end{array}$ & $\begin{array}{l}\text { Trauma and } \\
\text { ortho-pedic } \\
\text { ward (1) }\end{array}$ & $\begin{array}{l}\text { Elective and } \\
\text { trauma } \\
\text { patients, } \\
>48 \text { hrs in } \\
\text { hospital }\end{array}$ & $559 / 323$ & $\begin{array}{l}\text { Within 24- } \\
48 \text { hrs of } \\
\text { admission }\end{array}$ & $\begin{array}{l}\text { Nose, } \\
\text { perineum }\end{array}$ & $78(C)$ & 24.0 & & & & $\begin{array}{l}\text { Fair } \\
1 Y ; 2 N ; \\
3 Y ; 4 Y ; \\
5 Y ; 6 ? ; \\
7 N\end{array}$ \\
\hline $\begin{array}{l}11 / \\
2\end{array}$ & $\begin{array}{l}\text { Woltering } \\
{[45]} \\
\text { Germany } \\
2008\end{array}$ & & $\begin{array}{l}\mathrm{GH}(5), \\
\mathrm{RC}(3)\end{array}$ & Patients & $1.321 / 818$ & $\begin{array}{l}\text { On } \\
\text { a 2-days } \\
\text { screening } \\
\text { period in } \\
\text { each unit }\end{array}$ & $\begin{array}{l}\text { Nose, throat, } \\
\text { wounds }\end{array}$ & $\begin{array}{l}\text { GH: } 17(\mathrm{C}) \\
\mathrm{RC}: 6(\mathrm{C})\end{array}$ & $\begin{array}{l}\text { GH: } 3.4 \\
(2.1-5.6) \\
\text { RC: } 1.2 \\
(0.4-3.3)\end{array}$ & $\begin{array}{l}\text { GH: } 11.6 \\
\text { RC: } 5.6\end{array}$ & & & $\begin{array}{l}\text { Good } \\
1 N ; 2 Y ; \\
3 Y ; 4 Y ; \\
5 Y ; 6 N ; \\
7 Y\end{array}$ \\
\hline
\end{tabular}

: $\mathrm{UH}=$ University hospital, ICU = Intensive care unit, $\mathrm{TH}=$ Teaching hospital, $\mathrm{GH}=$ General hospital, $\mathrm{RC}=$ Rehabilitation centre, $\mathrm{LF}=$ Laboratory facility, n.r. $=$ not reported

2: Pooled mean calculated as a combined prevalence rate over all units with $95 \% \mathrm{Cl}$.

3 : Range within the individual units.

: Levels for study quality: Good (= 6 and 7 points), fair (= $3-5$ points), poor (= 1 and 2 points). Numbers belong to the questions as illustrated in Table 1 .

5: Specimens (blood, sputum, others) taken for diagnostic purposes. Abbreviations: $\mathrm{Cl}$, Confidence intervals; hrs, hours; N, no; wk, week; $\mathrm{Y}$, Yes; ?, unclear or missing information. 
characteristics (countries with known higher MRSA rates), but may also be caused by methodological weaknesses, as seen by the quality of the included studies. Interpretation of the results is most hampered by the heterogeneity in the study population, screening policy, study period, and calculation of the outcome.

\section{Study population}

As study participants were not homogenous, but were members of different populations (admissions, patients, residents, and $S$. aureus isolates), it was difficult to compare MRSA rates. In the clinical setting, inclusion criteria were time-related (on admission or during hospital stay) and risk-related (patients with a high risk for MRSA carrier status). Regardless of the different inclusion criteria, all studies are prone to underestimate the MRSA reservoir. Admission-only screening does not assess healthcare-associated transmission [13], but rather provides data on imported MRSA cases [28], although transmission of MRSA in most cases happened during a previous hospital stay. Selective screening of high-risk patients on admission to the hospital or to special wards is a strategy recommended by national guidelines, but risk factors for MRSA carriage are not standardized, nor were they described by the studies. In addition, poor compliance with selective risk factor based screening has been reported [29], so that not all colonized patients may have been detected. In the case of screening programs including only groups of highrisk patients after admission to hospital, MRSA rates are not representative for the hospitalized population as a whole $[13,14]$. Underestimation of the MRSA reservoir is also expected from studies relying exclusively on clinical cultures taken for therapeutic reasons, as they may fail to identify $85 \%$ of the MRSA-colonized patients [25].

In the LTC setting, even if all study participants were recruited as residents of nursing homes for the elderly, comparison of the MRSA rates is difficult, because potentially eligible cases were not described by inclusion criteria. The findings of this review suggest that screening programs in LTC facilities are not assigned to admission of new residents. However, time-points for screening were used in some studies in an almost standardized way (No.1,4,6,9,11), so that these rates may present representative rates for the population of residents in the nursing home as a whole. No statement is possible for endemic MRSA in LTC settings such as home care, day-care institutions for (multiply) handicapped people, or institutions for patients with longterm artificial respiration. Only one study (No.21) investigated the MRSA clearance on discharge from hospital of home-care patients colonized with MRSA.

\section{Screening policy}

Swabs were the preferred screening method in the included studies, but heterogeneity was found for the anatomical sites which had been swabbed. Even though the contribution of variations in sampling procedures is not clear [30], lack of sensitivity of the swabbing sites has to be considered when surveillance data from different facilities are compared [31]. For the studies using only nasal swabs, underreporting of MRSA cases is suggested, given that cultures of the nares identify only $60 \%-73 \%$ of the S. aureus carriers $[20,22,31,32]$. That is why in national guidelines screening of additional sites is recommended, though no consensus has been reached [4,31]. For reasons of accessibility, compliance and consistency with other investigations, it is recommended by Hori [14] that the investigation of MRSA prevalence should be confined to nasal swabs. Though this recommendation might be considered excessively strict, it is nevertheless reasonable, as it permits comparison between different studies.

The swabbing procedure is described in more detail in only a very few studies. Therefore, difference in swabbing procedures might also be a reason for the different MRSA rates. For standardization of MRSA measurement by surveillance cultures, the sampling procedures have to be considered [4] and it is recommended that the swab specimens are obtained only by specially trained healthcare workers [20].

\section{Study period}

The range in study period, between less than one months and six years, is due to the two most often used screening modes, point prevalence and prospective screening over different study phases. As the data were generally related to total study period, results are not comparable. According to the ORION statement, infection-related outcomes should be related to regular time units rather than presented as totals for study phase [18]. There are no standards for the most effective screening mode for MRSA prevalence. Even if pointprevalence rates offer the best choice for comparison, the limitations of this method should be kept in mind, as with point-prevalence only a short-time cut-out is considered and prevalence on another day might differ [33]. Repeated point-prevalence measurements are therefore recommended in order to achieve a more comprehensive view of the endemic situation [34].

\section{Calculation of the outcome}

Comparison of MRSA rates was hampered, as two different prevalence numerators were used, patients colonized with MRSA and methicillin-resistant isolates. A further difficulty was that the method of counting differed with regard to repeated admission or repeated MRSA-findings of a patient during the study period, which complicates the comparison.

Comparison of MRSA rates was also hampered, by the fact that the outcomes were reported either in the form 
of proportions or as prevalence density. For resistance proportions (expressed as MRSA among S. aureus isolates), consistency was observed for the clinical setting (between 13\% and 20\%), but these data are not available for the LTC setting. From a public health perspective, resistance proportions do not allow an unbiased estimate of the MRSA burden in the respective setting [35], and therefore density rates are recommended as a more appropriate measure for the average MRSA burden $[7,27]$. MRSA rates in the form of density data were presented by only a few studies. As most studies misleadingly reported incidence density using total patient days as denominators (instead of patient days at risk for new MRSA detection), the true incidence of MRSA acquisition is probably underestimated [13]. Concerning the differences in calculations of MRSA outcomes, the findings of our review are in line with the assessment of other authors, that up to now no surveillance method allowing calculation of the rate of MRSA colonization and infection has been gained acceptance as a valid method for comparisons between institutions [7].

One of the limitations in our review is that a high proportion of the retrieved articles was excluded from the review, as MRSA prevalence was not the primary objective. Inflation of MRSA hits is probably caused by a trend to add MRSA to both keyword lists and abstracts of every study of even peripheral relevance to the surveillance of antimicrobial resistance. Another limitation in our review is that the quality of the studies was not assessed by a validated score. However, the majority of the selected quality criteria have already been used to critically appraise research articles which estimate the prevalence of a disease [15].

\section{Conclusions}

Since the recognition of MRSA as a hospital problem largely depends on swabs or clinical samples taken on admission or during hospital-stay of high-risk patients, the true case load of a hospital remains largely unknown. In order to enable comparison between different studies and different settings, MRSA rates should be assessed in a standardized way, e.g. the anterior nares should always be included and sampling should be performed by a trained person.

Accurate incidence measures using denominator data adjusted for the at-risk population are warranted for comparing MRSA studies. However, these incidence measures are difficult to obtain, especially in LTC settings. Therefore less demanding estimates of MRSA rates should be used in a standardized way in clinical and LTC settings in order to allow comparison. Consensus should be achieved, in order to define standardized procedures for MRSA surveillance in different healthcare settings.

\section{Funding}

The study was funded by the Institution for Statutory Accident Insurance and Prevention in the Health and Welfare Services (BGW).

\section{Author details}

${ }^{1}$ Institution for Statutory Accident Insurance and Prevention in the Health and Welfare Services. Department of Occupational Health Research, Pappelallee 33/35/37, 22089 Hamburg, Germany. ${ }^{2}$ University Medical Center Hamburg-Eppendorf, Institute for Health Services Research in Dermatology and Nursing, Martinistr. 52, 20246 Hamburg, Germany.

\section{Authors' contributions}

MD performed the literature search, performed the extraction of the data, and drafted the initial manuscript; FH helped with data extraction and helped with data interpretation; CP and AS performed the quality scoring and helped with data interpretation; AN critically revised the manuscript for important content. All authors read and approved the final manuscript.

\section{Competing interests}

The authors declare that they have no competing interests.

Received: 14 January 2011 Accepted: 20 May 2011

Published: 20 May 2011

\section{References}

1. Lowy FD: Staphylococcus aureus infections. N Engl J Med 1998, 339:520-532.

2. Witte W, Kresken M, Braulke C, Cuny C: Increasing incidence and widespread dissemination of methicillin-resistant Staphylococcus aureus (MRSA) in hospitals in central Europe, with special reference to German hospitals. Clin Microbiol Infect 1997, 3:414-422.

3. Tiemersma EW, Bronzwaer SL, Lyytikäinen O, Degener JE, Schrijnemakers $P$, Bruinsma N, Monen J, Witte W, Grundmann H: Methicillin-resistant Staphylococcus aureus in Europe, 1999-2002. Emerg Infect Dis 2004, 10:1627-1634

4. Muto CA: Methicillin-resistant Staphylococcus aureus control: we didn't start the fire, but it's time to put it out. Infect Control Hosp Epidemiol 2006, 27:111-115.

5. Albrich WC, Harbarth S: Health-care workers: source, vector, or victim of MRSA? Lancet Infect Dis 2008, 8:289-301.

6. Haamann F, Dulon M, Nienhaus A: MRSA as an occupational disease: a case series. Int Arch Occup Environ Health 2011, 84:259-266.

7. Chaberny IF, Sohr D, Ruden H, Gastmeier P: Development of a surveillance system for methicillin-resistant Staphylococcus aureus in German hospitals. Infect Control Hosp Epidemiol 2007, 28:446-452.

8. Marshall C, Wesselingh S, McDonald M, Spelman D: Control of endemic MRSA-what is the evidence? A personal view. J Hosp Infect 2004, 56:253-268.

9. Muto CA, Jernigan JA, Ostrowsky BE, Richet HM, Jarvis WR, Boyce JM, Farr BM: SHEA guideline for preventing nosocomial transmission of multidrug-resistant strains of Staphylococcus aureus and enterococcus. Infect Control Hosp Epidemiol 2003, 24:362-386.

10. Simon A, Exner M, Kramer A, Engelhart S: Umsetzung der MRSAEmpfehlung der KRINKO von 1999 - Aktuelle Hinweise des Vorstandes der DGHK. Hyg Med 2009, 34:90-101.

11. KRINKO: Empfehlung zur Prävention und Kontrolle von Methicillinresistenten Staphylococcus-aureus-Stämmen (MRSA) in Krankenhäusern und anderen medizinischen Einrichtungen - Mitteilung der Kommission für Krankenhaushygiene und Infektionsprävention am Robert-KochInstitut. Bundesgesungheitsbl Gesunndheitsforsch Gesundheitsschutz 1999, 42:954-958.

12. Andersen BM, Rasch M, Syversen G: Is an increase of MRSA in Oslo, Norway, associated with changed infection control policy? J Infect 2007, 55:531-538.

13. Huang SS, Rifas-Shiman SL, Warren DK, Fraser VJ, Climo MW, Wong ES, Cosgrove SE, Perl TM, Pottinger JM, Herwaldt LA, Jernigan JA, Tokars JL, Diekema DJ, Hinrichsen VL, Yokoe DS, Platt R: Improving methicillin- 
resistant Staphylococcus aureus surveillance and reporting in intensive care units. J Infect Dis 2007, 195:330-338.

14. Hori S, Sunley R, Tami A, Grundmann H: The Nottingham Staphylococcus aureus population study: prevalence of MRSA among the elderly in a university hospital. J Hosp Infect 2002, 50:25-29.

15. Loney PL, Chambers LW, Bennett KJ, Roberts JG, Stratford PW: Critical appraisal of the health research literature: prevalence or incidence of a health problem. Chronic Dis Can 1998, 19:170-176.

16. Slim K, Nini E, Forestier D, Kwiatkowski F, Panis Y, Chipponi J: Methodological index for non-randomized studies (minors): development and validation of a new instrument. ANZ J Surg 2003, 73:712-716.

17. Des Jarlais DC, Lyles C, Crepaz N: Improving the reporting quality of nonrandomized evaluations of behavioral and public health interventions: the TREND statement. Am J Public Health 2004, 94:361-366.

18. Stone SP, Cooper BS, Kibbler CC, Cookson BD, Roberts JA, Medley GF, Duckworth G, Lai R, Ebrahim S, Brown EM, Wiffen PJ, Davey PG: The ORION statement: guidelines for transparent reporting of outbreak reports and intervention studies of nosocomial infection. J Antimicrob Chemother 2007, 59:833-840.

19. von Elm E, Altman DG, Egger M, Pocock SJ, Gotzsche PC, Vandenbroucke JP: The Strengthening the Reporting of Observational Studies in Epidemiology (STROBE) statement: guidelines for reporting observational studies. Epidemiology 2007, 18:800-804.

20. Mertz D, Frei R, Jaussi B, Tietz A, Stebler C, Fluckiger U, Widmer AF: Throat swabs are necessary to reliably detect carriers of Staphylococcus aureus. Clin Infect Dis 2007, 45:475-477.

21. Kluytmans J, van Belkum A, Verbrugh H: Nasal carriage of Staphylococcus aureus: epidemiology, underlying mechanisms, and associated risks. Clin Microbiol Rev 1997, 10:505-520.

22. Batra R, Eziefula AC, Wyncoll D, Edgeworth J: Throat and rectal swabs may have an important role in MRSA screening of critically ill patients. Intensive Care Med 2008, 34:1703-1706.

23. Kramer A, Wagenvoort H, Ahrén C, Daniels-Haardt I, Hartemann P, Kobayashi H, Kurcz A, Picazo J, Privitera G, Assadian O: Epidemiology of MRSA and current strategies in Europe and Japan. GMS Krankenhhyg Interdiszip 2010, 5(1):Doc01.

24. Coia JE, Duckworth GJ, Edwards DI, Farrington M, Fry C, Humphreys H, Mallaghan C, Tucker DR: Guidelines for the control and prevention of meticillin-resistant Staphylococcus aureus (MRSA) in healthcare facilities. $J$ Hosp Infect 2006, 63(Suppl 1):S1-44.

25. Salgado CD, Farr BM: What proportion of hospital patients colonized with methicillin-resistant Staphylococcus aureus are identified by clinical microbiological cultures? Infect Control Hosp Epidemiol 2006, 27:116-121.

26. Kresken M, Hafner D, Schmitz FJ, Wichelhaus TA, für die Studiengruppe: Resistenzsituation bei klinisch wichtigen Infektionserregern gegenüber Antibiotika in Deutschland und im mitteleuropäischen Raum. Bericht über die Ergebnisse einer multizentrischen Studie der Arbeitsgemeinschaft Empfindlichkeitsprüfungen \& Resistenz der Paul-Ehrlich-Gesellschaft fur Chemotherapie e.V. aus dem Jahre 2007 Rheinbach: Antiinfectives Intelligence; 2009.

27. Meyer $E$, Gastmeier $P$, Schwab F: The burden of multiresistant bacteria in German intensive care units. J Antimicrob Chemother 2008, 62:1474-1476.

28. Salgado CD, Vos MC, Farr BM: Universal screening for methicillin-resistant Staphylococcus aureus by hospitals. JAMA 2008, 300:503-504.

29. Gopal Rao G, Michalczyk P, Nayeem N, Walker G, Wigmore L: Prevalence and risk factors for meticillin-resistant Staphylococcus aureus in adult emergency admissions-a case for screening all patients? J Hosp Infect 2007, 66:15-21

30. Bloemendaal AL, Fluit AC, Jansen WM, Vriens MR, Ferry T, Argaud L, Amorim JM, Resende AC, Pascual A, Lopez-Cerero L, Stefani S, Castiglione G, Evangelopoulou P, Tsiplakou S, Rinkes $\mathrm{H}_{\text {, Verhoef J: }}$ Acquisition and cross-transmission of Staphylococcus aureus in European intensive care units. Infect Control Hosp Epidemiol 2009, 30:117-124.

31. Eveillard M, de LA, Lancien E, Barnaud G, Ricard JD, Joly-Guillou ML: Evaluation of a strategy of screening multiple anatomical sites for methicillin-resistant Staphylococcus aureus at admission to a teaching hospital. Infect Control Hosp Epidemiol 2006, 27:181-184.

32. Nilsson P, Ripa T: Staphylococcus aureus throat colonization is more frequent than colonization in the anterior nares. J Clin Microbiol 2006, 44:3334-3339.
33. Denis O, Jans B, Deplano A, Nonhoff C, De RR, Suetens C, Struelens MJ: Epidemiology of methicillin-resistant Staphylococcus aureus (MRSA) among residents of nursing homes in Belgium. J Antimicrob Chemother 2009, 64:1299-1306.

34. Chaberny IF, Wriggers A, Behnke M, Gastmeier P: Antibiotics: MRSA prevention measures in German hospitals: results of a survey among hospitals, perfrmed as part of the MRSA-KISS module. Dtsch Arztebl Int 2010, 107:631-637.

35. Schwaber MJ, De-Medina T, Carmeli Y: Epidemiological interpretation of antibiotic resistance studies - what are we missing? Nat Rev Microbiol 2004, 2:979-983.

36. Baldwin NS, Gilpin DF, Hughes CM, Kearney MP, Gardiner DA, Cardwell C, Tunney MM: Prevalence of methicillin-resistant Staphylococcus aureus colonization in residents and staff in nursing homes in Northern Ireland. J Am Geriatr Soc 2009, 57:620-626.

37. von Baum H, Schmidt C, Svoboda D, Bock-Hensley O, Wendt C: Risk factors for methicillin-resistant Staphylococcus aureus carriage in residents of German nursing homes. Infect Control Hosp Epidemiol 2002, 23:511-515.

38. Barr B, Wilcox MH, Brady A, Parnell P, Darby B, Tompkins D: Prevalence of methicillin-resistant Staphylococcus aureus colonization among older residents of care homes in the United Kingdom. Infect Control Hosp Epidemiol 2007, 28:853-859.

39. Brugnaro P, Fedeli U, Pellizzer G, Buonfrate D, Rassu M, Boldrin C, Parisi SG, Grossato A, Palu G, Spolaore P: Clustering and risk factors of methicillinresistant Staphylococcus aureus carriage in two Italian long-term care facilities. Infection 2009, 37:216-221.

40. Cretnik TZ, Vovko P, Retelj M, Jutersek B, Harlander T, Kolman J, Gubina M: Prevalence and nosocomial spread of methicillin-resistant Staphylococcus aureus in a long-term-care facility in Slovenia. Infect Control Hosp Epidemiol 2005, 26:184-190.

41. Heuck D, Fell D, Hamouda O, Claus H, Witte W: Erste Ergebnisse einer überregionalen Studie zur MRSA-Besiedlung bei Bewohnern von Altenund Pflegeheimen. Hyg Med 2000, 25:191-192.

42. Heudorf U, Bremer V, Heuck D: Methicillin-resistant Staphylococcus aureus in Long-term Care Facilities for the Aged in Frankfurt am Main, Germany, in 1999. Gesundheitswesen 2001, 63:447-454.

43. Hoefnagels-Schuermans A, Niclaes L, Buntinx F, Suetens C, Jans B, Verhaegen J, Van EJ: Molecular epidemiology of methicillin-resistant Staphylococcus aureus in nursing homes: a cross-sectional study. Infect Control Hosp Epidemiol 2002, 23:546-549.

44. Neuhaus B, Bocter N, Braulke C, Heuck C, Witte W: Survey of methicillinresistant Staphylococcus aureus in long-term facilities for the aged in North Rhine Westphalia. Bundesgesungheitsbl Gesunndheitsforsch Gesundheitsschutz 2002, 45:894-904.

45. Woltering R, Hoffmann G, Daniels-Haardt I, Gastmeier P, Chaberny IF: Prevalence of methicillin-resistant Staphylococcus aureus (MRSA) in patients in long-term care in hospitals, rehabilitation centers and nursing homes of a rural district in Germany. Dtsch Med Wochenschr 2008, 133:999-1003.

46. Chaberny IF, Bindseil A, Sohr D, Gastmeier P: A point-prevalence study for MRSA in a German university hospital to identify patients at risk and to evaluate an established admission screening procedure. Infection 2008, 36:526-532.

47. Chaberny IF, Ziesing S, Mattner F, Barwolff S, Brandt C, Eckmanns T, Ruden H, Sohr D, Weist K, Gastmeier P: The burden of MRSA in four German university hospitals. Int J Hyg Environ Health 2005, 208:447-453.

48. Eveillard M, Ernst C, Cuviller S, Lescure FX, Malpaux M, Defouilloy I, Gresanleux M, Duboisset M, Lienard J, Eb F: Prevalence of methicillinresistant Staphylococcus aureus carriage at the time of admission in two acute geriatric wards. J Hosp Infect 2002, 50:122-126.

49. Anonymous: Flächendeckendes einmonatiges MRSA-Prävalenzscreening in Akut- und Rehakliniken - Umsetzung in einem Flächenkreis außerhalb des EUREGIO-Netzwerks. Hyg Med 2010, 35:223-225.

50. Hassan K, Paturi A, Hughes C, Giles S: The prevalence of methicillin resistant Staphylococus aureus in orthopaedics in a non-selective screening policy. Surgeon 2008, 6:201-203.

51. Kappstein I, van der MK, Meschzan D, Vatou V, Bieg-Habermann S: Prevention of transmission of methicillin-resistant Staphylococcus aureus (MRSA) infection: standard precautions instead of isolation: A 6-year surveillance in a University hospital. Chirurg 2009, 80:49-61. 
52. Lucet JC, Paoletti X, Demontpion C, Degrave M, Vanjak D, Vincent C, Andremont A, Jarlier V, Mentre F, Nicolas-Chanoine MH: Carriage of methicillin-resistant Staphylococcus aureus in home care settings: prevalence, duration, and transmission to household members. Arch Intern Med 2009, 169:1372-1378.

53. Lucet JC, Grenet K, Armand-Lefevre L, Harnal M, Bouvet E, Regnier B, Andremont A: High prevalence of carriage of methicillin-resistant Staphylococcus aureus at hospital admission in elderly patients: implications for infection control strategies. Infect Control Hosp Epidemiol 2005, 26:121-126.

54. Meyer E, Schwab F, Gastmeier P, Rueden H, Daschner FD: Surveillance of antimicrobial use and antimicrobial resistance in German intensive care units (SARI): a summary of the data from 2001 through 2004. Infection 2006, 34:303-309.

55. Morange-Saussier V, Giraudeau B, van der Mee N, Lermusiaux P, Quentin R: Nasal carriage of methicillin-resistant staphylococcus aureus in vascular surgery. Ann Vasc Surg 2006, 20:767-772.

56. Reilly JS, Stewart S, Christie P, Allardice G, Smith A, Masterton R, Gould IM, Williams C: Universal screening for meticillin-resistant Staphylococcus aureus: interim results from the NHS Scotland pathfinder project. J Hosp Infect 2010, 74:35-41.

57. Tai CC, Nirvani AA, Holmes A, Hughes SP: Methicillin-resistant Staphylococcus aureus in orthopaedic surgery. Int Orthop 2004, 28:32-35

58. Thompson DS: Methicillin-resistant Staphylococcus aureus in a general intensive care unit. J R Soc Med 2004, 97:521-526.

59. Vos MC, Behrendt MD, Melles DC, Mollema FP, de GW, Parlevliet G, Ott A, Horst-Kreft D, van BA, Verbrugh HA: 5 years of experience implementing a methicillin-resistant Staphylococcus aureus search and destroy policy at the largest university medical center in the Netherlands. Infect Control Hosp Epidemiol 2009, 30:977-984.

60. Walley G, Orendi J, Bridgman S, Davis B, Ahmed el, Maffulli N: Methicillin resistant Staphylococcus aureus (MRSA) is not always caught on the orthopaedic ward. Acta Orthop Belg 2009, 75:245-251.

\section{Pre-publication history}

The pre-publication history for this paper can be accessed here: http://www.biomedcentral.com/1471-2334/11/138/prepub

doi:10.1186/1471-2334-11-138

Cite this article as: Dulon et al:: MRSA prevalence in european healthcare settings: a review. BMC Infectious Diseases 2011 11:138.

\section{Submit your next manuscript to BioMed Central and take full advantage of:}

- Convenient online submission

- Thorough peer review

- No space constraints or color figure charges

- Immediate publication on acceptance

- Inclusion in PubMed, CAS, Scopus and Google Scholar

- Research which is freely available for redistribution

Submit your manuscript at www.biomedcentral.com/submit 